\title{
An Alternate Methodology for Studying Diffusion and Elution Kinetics of Dimethacrylate Monomers Through Dentinal Tubules
}

\section{Dol:}

10.1016/j.dental.2020.02.008

\section{Document Version}

Accepted author manuscript

Link to publication record in Manchester Research Explorer

Citation for published version (APA):

Durner, J., Schrickel, K., Watts, D. C., Becker, M., Hickel, R., \& Draenert, M. E. (2020). An Alternate Methodology for Studying Diffusion and Elution Kinetics of Dimethacrylate Monomers Through Dentinal Tubules. Dental Materials. https://doi.org/10.1016/j.dental.2020.02.008

\section{Published in:}

Dental Materials

\section{Citing this paper}

Please note that where the full-text provided on Manchester Research Explorer is the Author Accepted Manuscript or Proof version this may differ from the final Published version. If citing, it is advised that you check and use the publisher's definitive version.

\section{General rights}

Copyright and moral rights for the publications made accessible in the Research Explorer are retained by the authors and/or other copyright owners and it is a condition of accessing publications that users recognise and abide by the legal requirements associated with these rights.

\section{Takedown policy}

If you believe that this document breaches copyright please refer to the University of Manchester's Takedown Procedures [http://man.ac.uk/04Y6Bo] or contact uml.scholarlycommunications@manchester.ac.uk providing relevant details, so we can investigate your claim.

\section{OPEN ACCESS}




\title{
An Alternate Methodology for Studying Diffusion and Elution Kinetics of Dimethacrylate Monomers Through Dentinal Tubules
}

\author{
Jürgen Durner ${ }^{* 1,2}$, Klaus Schrickel ${ }^{3}$, David C. Watts ${ }^{4}$, Marc Becker ${ }^{1,2}$, Reinhard Hickel ${ }^{1}$, \\ Miriam E. Draenert ${ }^{1}$
}

${ }^{1}$ Department of Conservative Dentistry and Periodontology, University Hospital, LMU

Munich Ludwig-Maximilians-University of Munich, Goethestr. 70, 80336 Munich, Germany

${ }^{2}$ Laboratory Becker and colleagues, Führichstr. 70, 81671 München, Germany

${ }^{3}$ Thermo Fisher Scientific, Im Steingrund 4 - 6, 63303 Dreieich, Germany

${ }^{4}$ School of Medical Sciences and Photon Science Institute, University of Manchester, Oxford Road, Manchester M13 9PL, United Kingdom

*Corresponding author:

Prof. Dr. rer. nat. Dr. med. Jürgen Durner

Department of Conservative Dentistry and Periodontology, University Hospital

Ludwig-Maximilians-University Munich

Goethestraße 70

80336 Munich

Germany

Email: juergen.durner@med.uni-muenchen.de

Phone: +49-89-44005-9301

Fax: +49-89-44005-9302 


\section{Highlights}

1. An alternative methodology is described to study passive diffusion of residual monomers through the coronal dentinal tubules stimulated via eluent liquids surrounding the root structures only.

2. Measurements have been conducted at $37^{\circ} \mathrm{C}$ over a $7 \mathrm{~d}$ period and key variables including residual dentin thickness and eluent media have been considered.

3. GC/MS methods have been used to detect and quantify a range of dimethacrylate monomers in the eluent media and the kinetics of elution.

4. Critical periods post-restoration for exposure of different monomers to pulp cells have been identified.

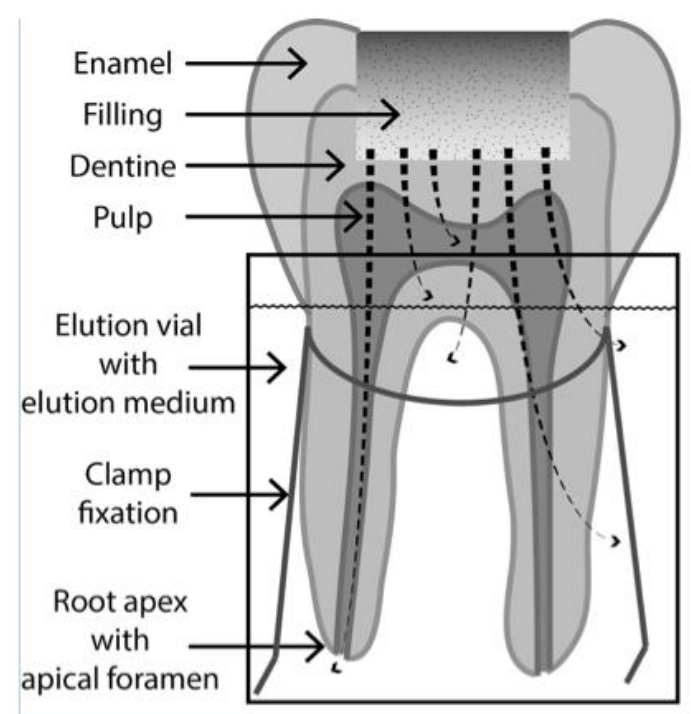




\begin{abstract}
Objectives. Ethoxylated bisphenol A dimethacrylate (bisEMA) is a base monomer in several dental resin composites. It was the main aim of the present study to determine if bisEMA can reach the dental pulp by generally passive diffusion through the coronal dentinal tubules stimulated via eluent liquids surrounding the root structures only.

Methods. In 20 human third molar teeth, standard Class-I occlusal cavities were prepared and provided either with an adhesive system alone or additionally with a composite restoration, according to the instructions of the manufacturer. The teeth were placed in an elution chamber such that the elution media only came into contact with the tooth root/tooth base where they were incubated at $37{ }^{\circ} \mathrm{C}$ for up to $7 \mathrm{~d}$. Samples were taken after 1, 2, 4 and $7 \mathrm{~d}$. Gas chromatography/mass spectrometry was used to identify bisEMA and other monomers in ethanol/water (3:1) and aqueous eluates.

Results. bisEMA was only found in ethanol/water eluates, where the teeth had received a composite restoration. Traces of bisEMA with up to three ethylene oxide units could be detected in these eluates. Depending on the dentin thickness, different elution kinetics of bisEMA were determined. Regardless of the treatment of teeth, triethylene glycol dimethacrylate (TEGDMA) and tetraethylene glycol dimethacrylate (TEEGDMA) were found in ethanolic/aqueous eluates in equal amounts. Most TEGDMA and TEEGDMA diffused through the dentin within the first $24 \mathrm{~h}$.

Significance. Depending on the dentin layer thickness, bisEMA was released for varied time periods, resulting in varied concentrations and exposure times for the different cells of the dental pulp. The concentrations of TEGDMA and TEEGDMA were greatest for cells of the dental pulp within the first $24 \mathrm{~h}$.
\end{abstract}

\title{
Keywords:
}

GC/MS, bisEMA; bisphenol A ethoxylate dimethacrylate; dentin barrier; dentinal tubules; elution; diffusion kinetics. 


\section{Introduction}

Over recent decades, the number and placement of methacrylate-based dental restorative materials has risen sharply. Methacrylic acid and its derivatives are a widely used substance class for dental monomers [1]. In addition to composite restorations they are also used in glass ionomer cements, resin-based luting composites, root fillings and adhesive systems as well as in pit and fissure sealants. The physical properties of methacrylic acid derivatives range from fluid to highly viscous liquids, from hydrophilic to lipophilic, from low to high molecular mass. Particularly widespread derivatives are low-viscosity and low-molecular mass monomers, mainly 2-hydroxyethyl methacrylate (HEMA), triethylene glycol dimethacrylate (TEGDMA) and/or tetraethylene glycol dimethacrylate (TEEGDMA), as well as the highviscous and high-molecular mass substances such as bisphenol A glycidyldimethacrylate (bisGMA) and ethoxylated bisphenol A dimethacrylate (bisEMA; bisphenol A ethoxylate dimethacrylate). In addition to these substances, which are largely responsible for the mechanical and physical properties of the restorative/bonding system, there are other components such as camphorquinone (CQ), which are important for photopolymerization. The compositions of methacrylate-based materials differ according to their function. Some of the monomers used have been detected in eluates (e.g. aqueous, methanolic, artificial saliva) after polymerization of the restorative material $[2,3]$.

This is possible because the degree of conversion (DC) of the carbon-carbon $(\mathrm{C}=\mathrm{C})$ double bond of the methacrylate-based monomers varies between 40 to $80 \%$ and therefore unreacted substances can be released $[4,5]$. It is well known that released monomers, co-monomers, initiators, stabilizers, decomposition-products or contaminants can lead in vitro to cytotoxic, teratogenic, estrogenic, mutagenic and/or genotoxic effects as well as to allergic reactions in vivo, such as allergic contact dermatitis and asthma in humans [6-11]. The DC decreases with increasing layer thickness towards depth [12]. This is explained by the fact that the light cannot penetrate so deeply and therefore less radicals are formed in the depth, which start the 
polymerization, which in turn leads to reduced conversion of the monomers to the polymer. Applied to a standard dental restoration, this means that light-induced polymerization is initiated via occlusal light delivery and the light penetration and resultant DC may decrease towards the pulp.

The released substances can be eluted by saliva and enter the gastrointestinal tract when swallowed, but can also reach the dental pulp by diffusion through the coronal dentinal tubuli. In vitro it was shown that uncured monomers leaching from composites can diffuse through dentin and reach the pulp within minutes after placement of a restorative material [13]. Within the dental pulp some of the substances can lead to a cytotoxic response. Thereby consequences may arise such as post-operative pain, pulpitis and in worst cases pulp necrosis $[5,14]$. The diffusion of substances through the dentin tubuli can be simulated by different models such as teeth in a chamber [15] or the use of dentin slices with variables such as dentin thickness, test setup, bovine or human teeth slices and further pretreatment of the teeth $[16,17]$. Dentin discs with a thickness of 300 to $500 \mu \mathrm{m}$ are often sawn from human teeth [5, 18]. These dentin discs are usually subjected, on one side, to the usual restorative preparation, as also performed in humans, and then placed in a pulp chamber model, where different simulations are performed. These include pure diffusion, after different exposure times to light, or diffusion using different elution media and/or additives to elution media. Usually the dentin discs have to be pretreated - often by removing the smear layer with $50 \%$ citric acid. The cutting process as well as the further processing afterwards could change the diffusion behavior, since the procedure could affect the dentin tubuli, by mechanical and/or thermal influences during sawing or via demineralisation by the strong chelator citric acid. In addition, the thickness of the dentin discs determines the diffusion distance, which can be significantly shorter than the physiological thickness of the dentin layer. Moreover, tubule diameters are greater closer to the pulp, so the outcome can depend upon the axial height at 
which the dentin disks are cut. The diffusion path could influence the amount and type of substances that can be eluted (e.g. low or high molecular mass substances, and hydrophilic or hydrophobic molecules).

To avoid the above-mentioned limitations, we have developed a model in which human teeth without further pretreatment are treated with a standardized cavity restoration as used in routine dental treatment of patients. The closeness to real patient care is a very important point for us. For this reason, we have chosen a standard Class I restoration of a tooth with a carious lesion. As in reality, a standard restoration can leave different residual dentin thicknesses.

The cavities can be restored with an adhesive system alone or with an adhesive system and a light-cured, commercially available composite. These teeth can be placed in a special test chamber, where the diffusion of substances/monomers through the dentinal tubuli into different elution media can be determined precisely since other surfaces are not in contact with the different elution media (Fig. 1). The focus of this investigation was on: (i) the detection in the eluate of high-molecular mass methacrylate-based monomers particularly bisEMA and its homologous (Fig. 2) indicating a diffusion through the residual dentin barrier and (ii) the determination of temporal diffusion kinetics as a function of dentin thickness and elution medium. Furthermore, the experimental setup can be used to estimate the proportion of monomers originating from the adhesive system and the proportion of monomers originating from the composite. Two different gas chromatography/mass spectrometry (GC/MS) methods were used: (a) for the determination of the higher boiling substances such as bisEMA and (b) for the lower boiling substances such as TEGDMA. 


\section{Methods}

All solvents and reagent products were obtained from Merck, Darmstadt, Germany and were of the highest purity available. The dental restorative materials used and their compositions are listed in Table 1.

\subsection{Tooth preparation}

20 extracted human third molar teeth were used and prepared for our examination. Ethics approval for the extracted molars was obtained by the Commission for Medical Ethics of the Ludwig-Maximilians-University, Munich (July 2018, No 18-551DE). The teeth selected fulfilled the following criteria: no dental caries or decay, no lesions and matured tooth growth. The teeth were stored after extraction and prior to treatment in Ringer's solution mixed with $0.1 \%$ sodium azide, without further preparation steps such as sterilization.

The preparation process was initiated after the teeth were rinsed under water for $10 \mathrm{~min}$. Standardized cylindrical Class I inlay cavities ( $3 \mathrm{~mm}$ long, $3 \mathrm{~mm}$ wide and $3 \mathrm{~mm}$ deep) were drilled in each tooth using a $151 \mu \mathrm{m}$ grain size diamond drill (Komet Dental Brasseler, Lemgo, Germany) for the initial preparation and a $46 \mu \mathrm{m}$ grain size drill (Komet Dental Brasseler) for the finishing surface at $40,000 \mathrm{rpm}$ and under constant water cooling. The treated surfaces were completely dried, corresponding to clinical drying using a rubber dam.

\subsection{Cavity treatment}

Each cavity was etched using a $37 \%$ phosphoric acid gel (Ivoclar Vivadent, Schaan, Liechtenstein). This was applied for $30 \mathrm{~s}$ on the enamel and for $15 \mathrm{~s}$ on the dentin surfaces. The phosphoric acid was thoroughly rinsed off with water and the surface dried using air flow.

10 teeth were treated using only the three-step adhesive system: Syntac ${ }^{\circ}$ Classic (Ivoclar Vivadent). The adhesive system was applied according to the Manufacturer's instructions. 
Briefly, a primer liquid was applied using a disposable brush for $15 \mathrm{~s}$ and carefully blown-off and dried. Subsequently an adhesive liquid was applied for $10 \mathrm{~s}$ and thoroughly dried in an air flow. Heliobond ${ }^{\circledR}$ (Ivoclar Vivadent), as a bonding layer, was applied and thinly dispersed. The adhesive system was cured for $10 \mathrm{~s}$ using a light-curing unit (Satelec $®$ Acteon $®$, Mini L.E.D. SN 304863181, Merignac Cedex, France) with an output irradiance of $1000 \mathrm{~mW} / \mathrm{cm}^{2}$. 10 further teeth were treated with the adhesive system (as above) and the cavities were filled with the composite: Tetric EvoCeram ${ }^{\circledR}$ (Ivoclar Vivadent). The cavities were filled via a layering technique according to the manufacturer's instructions (max. $2 \mathrm{~mm}$ layer), whereby each layer was cured for 20 s. using a light-curing unit (Satelec $®$ Acteon $®)$ with an irradiance of $1000 \mathrm{~mW} / \mathrm{cm}^{2}$.

\subsection{Elution experiments}

After preparation, the teeth were assigned into different groups according to the elution medium (ethanol/water (3:1) or distilled water) and their dental treatment. Four groups with five teeth each were formed: EW-A: Teeth only treated with the adhesive system (A) were incubated in ethanol/water $(3: 1 ; \mathrm{EW})$; EW-AC: Teeth treated with the adhesive system and composite $(\mathrm{AC})$ were incubated in ethanol/water $(3: 1 ; \mathrm{EW})$; W-A: Teeth only treated with the adhesive system (A) were incubated in distilled water (W); W-AC: Teeth treated with the adhesive system and composite (AC) were incubated in distilled water (W). Subsequently, the group abbreviations were followed by the tooth number.

The teeth were mounted in $5 \mathrm{ml}$ glass vials (Wagner \& Munz, Munich, Germany) using a wire fixture that ensured that the root only would be completely immersed in the elution medium, while the crown remained above the surface of the elution medium. The fixture was made of a dental wire wound around the tooth neck at the enamel border (Fig. 1).

Then the elution medium was added. Depending on the size of the tooth, between 3.5 and 4.5 $\mathrm{ml}$ of the elution liquids were added to reach the required level up to the crown of the tooth 
(see paragraph above). Then the glass vials were sealed and incubated at $37{ }^{\circ} \mathrm{C}$. After $1,2,4$ and $7 \mathrm{~d}, 100 \mu \mathrm{l}$ from each specimen vial were taken and stored in GC-vials (Thermo Fisher Scientific, Waltham, Massachusetts, USA). The removed liquids were replaced with the same volume of either ethanol/water (3:1) or distilled water.

The diffusion of the leached monomers depended on the thickness of the residual dentin. Therefore, it was determined. The shortest distance between the bottom of the cavity and the pulp chamber was measured by X-ray images $(10 \mathrm{~cm}$ distance, $60 \mathrm{kV}$ at $0.08 \mathrm{sec}$, Sirona Dental Systems, Wals, Austria; ModellTyp: No 3343741). A 20 mm endodontic file (Fa VDW GmbH Munich, Germany; Typ: K-Feile-ISO 30) was used as the reference scale.

\subsection{GC/MS analysis}

The analysis of the eluates was performed on a Thermo Scientific ${ }^{\text {TM }}$ TRACE $^{\text {TM }} 1310$ gas chromatograph connected to a Thermo Scientific ${ }^{\mathrm{TM}} \mathrm{ISQ}^{\mathrm{TM}} 7000$ mass spectrometer (Thermo Fisher Scientific). Analysis was conducted using two different GC/MS methods. For reasons of comparability, a standard method was applied. A second method, working at higher temperatures and with, necessarily, a suitable GC column, was used to increase the recovery of higher boiling components. With the first method "normal" substances like TEGDMA and TEEGDMA were determined. A Thermo Scientific ${ }^{\mathrm{TM}}$ TraceGOLD ${ }^{\mathrm{TM}}$ TG5Silms capillary column (length $30 \mathrm{~m}$, inner diameter $0.25 \mathrm{~mm}$, coating $0.25 \mu \mathrm{m}$; Thermo Fisher Scientific) was used as the capillary column for GC separations under standard operating conditions. The GC oven was heated from $50{ }^{\circ} \mathrm{C}$ ( 2 min isotherm) to $300{ }^{\circ} \mathrm{C}$ (5 min isotherm) with a rate of $10{ }^{\circ} \mathrm{C} / \mathrm{min}$ and $1.0 \mu \mathrm{l}$ of the solution was injected via a programmed temperature vaporization (PTV) injector. Helium was used as carrier gas at a constant flow rate of $1.0 \mathrm{ml} / \mathrm{min}$. The temperature of the PTV injector was $50{ }^{\circ} \mathrm{C}$ during the injection phase and then increased to $300{ }^{\circ} \mathrm{C}$ with a rate of $14{ }^{\circ} \mathrm{C} / \mathrm{s}$. The temperature of the direct coupling (transfer line) to the MS 
was $300{ }^{\circ} \mathrm{C}$. The MS was operated in electron ionisation mode (EI, $\left.70 \mathrm{eV}\right)$, with the ion source operated at $300{ }^{\circ} \mathrm{C}$; only positive ions were scanned. Scans were made over the range $\mathrm{m} / \mathrm{z} 40-600$ at a scan rate of $5 \mathrm{scan} / \mathrm{s}$ for scans operated in full scan mode for identification. Caffeine was used as internal standard.

An additional analysis (second method for the determination of bisGMA as well as bisEMA and its homologous) at higher temperatures was done using a TraceGOLD TG-XLBms column, $20 \mathrm{~m}$ x $0.18 \mathrm{~mm}$ ID x $0.18 \mu \mathrm{m}$ FT (Thermo Fisher Scientific). The GC oven was programmed from $50{ }^{\circ} \mathrm{C}$ for $1 \mathrm{~min}$ to $330{ }^{\circ} \mathrm{C}$ for $5 \mathrm{~min}$ at a rate of $15^{\circ} \mathrm{C} / \mathrm{min}$. PTV injection $(1.0 \mu \mathrm{l})$ was done at $50{ }^{\circ} \mathrm{C}$ increasing to $350{ }^{\circ} \mathrm{C}$ with a rate of $14{ }^{\circ} \mathrm{C} / \mathrm{s}$. The MS transfer line and MS ion source were heated to $320^{\circ} \mathrm{C}$. GC/MS system, carrier gas settings and scan range were the same as for the first method. In the single ion mode (SIM) targeted analysis of the bisEMA homologues was performed for the following m/z: 452, 496, 525, and 569 for quantitation of the analytes. In the case of the analytics of the dissolved composite in methanol the scans were made over a range $\mathrm{m} / \mathrm{z}, 100-800$ at a scan rate of $5 \mathrm{scan} / \mathrm{s}$.

The integration of the chromatograms was carried out over the base peak or other characteristic mass peaks of the compounds, typically $\mathrm{M}^{+}$and $\mathrm{M}^{+}-15$. Identification of the various substances was achieved by chemical analysis of their fragmentation patterns [19].

\subsection{Calculations and statistics}

The analytical results are presented as means. The standard deviation (SD) was calculated from the variation of the caffeine signal. The evaluation regarding the amount of an analyte was based on the peak area under the curve for each analyte. An alternative parameter was the peak height. Due to the different thickness of the remaining dentin, the quantities of an analyte found (area under the curve) were also related to the residual thickness. For appropriate cases, the measured quantity of the analyte was divided by the measured thickness 
of the residual dentin. For analytes where there was a significant progressive time-dependence of the released molecules, a non-linear regression analysis was used to model the elution kinetics. For this purpose, a 3-parameter single exponential function with an increase to a maximum, a standard function in physics for the description of such kinetic processes, was used.

$$
y=y_{0}+a *\left(1-e^{-k * t}\right)
$$

with $\mathrm{y}_{0}$ and $\mathrm{a}=$ modulation factors, $\mathrm{k}=$ growth constant, $\mathrm{t}=$ elution time [d].

For the analytes bisEMA or bisGMA the integrated signal areas (y) at each time-point were fitted to this equation. To determine the parameters $\mathrm{y}_{0}$, a and $\mathrm{k}$, the software program (SigmaPlot 12, Systat Software, Erkrath, Germany) uses a reduced chi-square algorithm. According to this algorithm, the parameters $\mathrm{y}_{0}$, a and $\mathrm{k}$ are adjusted in such a way that at the measuring points, from the integrated signal areas $\mathrm{y}$, to the resulting exponential function have the smallest mean square deviation. 


\section{Results}

The dentin thicknesses ranged from 0.83 to $3.51 \mathrm{~mm}$, mean value $1.89 \mathrm{~mm}$, standard deviation $(\mathrm{SD}) \pm 0.62$ (Table 2).

The analytical stability of the GC/MS method was determined by repeated measurement of caffeine added to the samples and was below $10 \%$.

\subsection{Aqueous elution and elution kinetics}

With the aqueous eluate W-A 1-5 and W-AC 1-5, TEGDMA and TEEGDMA were found in quantifiable amounts. The release of both comonomers showed an increase mostly within the first two days (Fig. 3). When the cavity was treated with a composite and an adhesive (W-AC 1-5) in contrast to an adhesive alone (W-A 1-5), greater amounts of TEGDMA and TEEGDMA were found. By dividing the amount of elutable monomers by the dentine thickness, it was evident that the amounts of TEGDMA and TEEGDMA in the aqueous eluates were independent of the dentin thickness. Thus an exponential release, according to equation 1 , was not found.

In the aqueous eluate $\mathrm{W}-\mathrm{A}$ 1-5 and $\mathrm{W}-\mathrm{AC}$ 1-5, neither bisEMA nor bisGMA was present in quantifiable amounts, although traces of these monomers could be detected.

\subsection{Ethanol/water elution and elution kinetics}

In the ethanol/water eluates from EW-A 1-5, TEGDMA and TEEGDMA were found in quantifiable amounts (Fig. 4). As measured by the integrated signal areas, more TEEGDMA then TEGDMA was found. However, after the first and until the seventh day no relevant changes in the amounts of either monomer in the eluate could be found. The main release of both monomers occurred within the first $24 \mathrm{~h}$. By dividing the amount of elutable monomers by the dentin thickness it was shown that, after treating the cavity with an adhesive alone 
(EW-A 1-5), the amounts of TEGDMA and TEEGDMA in the ethanol/water eluates were independent of the dentin thickness (Fig. 4).

In the ethanol/water eluates from EW-A 1-5, no bisEMA was found. Only in the ethanol/water eluates from EW-A 5 was bisGMA found.

In the ethanol/water eluates from EW-AC 1-5, TEGDMA and TEEGDMA were found in quantifiable amounts (Fig. 5). As measured by the integrated signal areas, more TEEGDMA then TEGDMA was found. After the first, and until the seventh day, no relevant changes in the amounts of either monomer in the eluate could be found. The main release of both monomers occurred within the first $24 \mathrm{~h}$. By dividing the amount of elutable monomers by the dentin thickness, it was shown that, after treating the cavity with an adhesive and a composite, the amount of TEGDMA and TEEGDMA in the ethanol/water eluates did show an indication of dependence on the dentin thickness (Fig. 5).

In the ethanol/water eluates from EW-AC 1-5 bisEMA was found. In these eluates only the bisEMA homologue with the lowest molecular weight was detectable $(m=1 ; n=1 ;$ Fig. 2$)$. Investigation of the composite dissolved in methanol showed that bisEMA homologues $n+m$ $\geq 4$ were not found in significant amounts, but traces were detected. The signal intensity (area) decreased the higher the sum of $n+m$.

By dividing the amount of elutable bisEMA by the dentin thickness, it was shown that less monomer could be detected in the elution medium with increasing dentin thickness in the same time interval (Fig. 6). This was recognized by the fact that a grouping depending on the dentin thickness seems possible (Fig. 6). This was also shown via determination of the kinetic growth constant $(\mathrm{k})$ by equation 1 . The growth constant $\mathrm{k}$ increased with increasing dentin thickness, related to the area: $\mathrm{k}(\mathrm{EW}-\mathrm{AC} 2)=0.49, \mathrm{k}(\mathrm{EW}-\mathrm{AC} 3)=0.37, \mathrm{k}(\mathrm{EW}-\mathrm{AC} 4)=0.21$ 
(Table 2). The elution kinetics of bisEMA, calculated according to equation 1, thus depended on the dentin thickness. 


\section{Discussion}

\subsection{The two main pathways for elution of monomers in vivo}

Many studies are concerned with the elution of substances from composite-based restorative materials, particularly after different treatments such as bleaching [20]. A large number of these studies use defined test specimens and place them in the elution medium and identify elutable substances, measure the amount of elutable substances or determine the elution kinetics from some substances. These experimental set-ups typically simulate the directly intra oral elution of substances into the oral cavity through saliva or (alcoholic) beverages. Fewer studies are concerned on the trans-dentinal diffusion via tubules. Thereby the substances diffuse through the dentin tubuli to the dental pulp. Several other experimental setups for simulating diffusion through the dentin layer are more complex (requiring production of dentin discs) compared to simulating the release of substances into the oral cavity.

\subsection{The potential adverse consequences of monomer elution}

According to the different release routes, the eluted substances come into contact with different types of cells. Substances eluted into the oral cavity come into contact with cells of the oral mucosa as well as with cells of the gastro-intestinal system through swallowing. Substances that reach the pulp trans-dentally can come into contact with the cells there. It is known that some elutable substances such as TEGDMA may have toxic effects on the dental pulp cells or its dental pulp stem cells [21, 22]. For this reason, determination of substances diffusing through dentin tubuli and the elution kinetics from these substances are of toxicological interest. 


\subsection{What precautions are necessary?}

Apart from the analytical fluctuations and pre-analytical disturbing factors, physiological differences (different dentin thickness) and possible structural changes of the dentin (pathogenic damage such as carious lesions) may affect the amount of elutable substances as well as the diffusion kinetics. To exclude some of these points, in our study, caries and restoration-free 3rd molars were used.

\subsection{How does this (alternative) tooth model differ from previous trans-tubular elution} studies?

Correspondence to real patient care is important. Therefore, a frequent mode of patient care in dental practice was simulated by restoration of a human tooth having a Class I cavity as would arise according to standard practice from removal of a carious lesion with the greatest possible preservation of healthy tooth substance. The thickness of the remaining dentin varies from person to person due to the physiological variation in dentin thickness. This is one reason why we have not chosen dentin discs of the same thickness, as in previous studies [5, 16]. Another reason is that the same dentin thickness does not necessarily mean that diffusion is standardized, since dentin tubules increase in both areal number density and in diameter as they get closer to the pulp [23, 24]. This does not include effects of other dental diseases [23, 24]. This should be considered when preparing dentin discs (either several from one tooth or from multiple teeth). If the dentin discs are prepared close to the pulp, the diameters are larger, which can change the amount of elutable substances, and also possibly allow larger molecules to diffuse, that would not diffuse equivalently via smaller diameters. Furthermore, some studies use bovine dentin. Depending on the layer and treatment used, this may differ significantly from human dentin in terms of hydraulic conductance and diffusional water flux [17]. Moreover, acid-treatment of the dentin discs is not necessary, as described in the introduction. A disadvantage of our model is the larger number of teeth that are required for 
statistical analysis. If thin dentin discs are used, the number of human teeth can be reduced as several discs per tooth can be produced as needed for statistical analysis.

\subsection{What were the elution media used? How did it reach the restorative materials?}

The quantities of eluted substances from the analysed materials also depends upon the elution medium used. To check whether lipophilic substances are also released, it has been shown that ethanol/water 3:1 is a more efficient elution medium than water or artificial saliva. Furthermore, ethanol/water 3:1 media is considered by the United States Food and Drug Administration (US FDA) as a food/oral simulating liquid of clinical relevance [25, 26]. Against this background we have chosen a strong elution medium (ethanol/water (3:1)) as well as a weaker medium (distilled water) for our elution experiments. Regardless of whether the teeth were treated with an adhesive only or with an adhesive and a composite, both elution media were used and consequently four groups resulted: EW-A, EW-AC, W-A, W-AC. In contrast to many other elution experiments [20], the composite restoration was not completely surrounded by the elution medium. In our experimental setup, the elution medium must penetrate the pulpal chamber mainly via the opening at the root tip. It then enters the dentinal tubules and reaches the restorative restoration.

\subsection{What physiological and physico-chemical factors influence diffusion and elution?}

Diffusion, in this context, depends on many factors; only the most important ones shall be mentioned. Firstly, there is the concentration gradient of a substance. Diffusional molecular flow from higher to lower concentrations is described by Fick's 1st law. Secondly, molecular size plays a role: smaller molecules often move faster than macromolecules. Thirdly, a higher viscosity of the elution medium reduces the diffusion rate. Fourthly, obstacles such as (cell) membranes influence diffusion. Fifthly, the overall diffusion time depends on the total distance to be covered [27]. 
Elution is often also called leachability. The influence of the elution medium is correspondingly significant. Its accessibility to the exchangeable substance (resin-composite) is also important. Both diffusion and elution depend on temperature and pressure. The points discussed above are based on 'pure' physic-chemical considerations. Diffusion and elution in dentistry can be additionally influenced by the chewing process and the hardness of food.

Camps et al. [28] prepared dentin discs with various adjacent composite fillings. These discs were placed in a pulp chamber and the diffusion of substances into a cell culture medium was determined. The toxicity of this mixture of elutable substances was then tested using cell cultures. In this test setup, the pulp pressure was also varied. One result was that the simulation of pulsatile pulp pressure increased cytotoxicity, which, conversely, would be compatible with increased diffusion of substances from the restoration.

In healthy teeth, enamel and dentin form a barrier, but are permeable to water and ions, i.e. diffusion from the tooth surface to the pulp and vice versa is possible [29]. Accordingly, the pulp pressure is not uniquely directed towards the oral cavity. One reason for this molecular flow is the concentration gradient of substances (Fick's 1st law). In a series of experiments with various drugs such as metronidazole, it was shown that in a split-chamber model with dentin discs, passive diffusion takes place at $15 \mathrm{~cm} \mathrm{H}_{2} \mathrm{O}$ pulpal pressure through the dentin (it was also shown that diffusion can be increased by iontophoresis) [30]. In this test arrangement, the chamber on the enamel side was filled with the drug dissolved in buffer, the chamber on the pulp side only with buffer, so that a concentration gradient was present. In another simulation, the influence of the chewing process as a function of the hardness of the food on the flow in the pulp was studied. Hard food causes a higher deformation of the tooth structure and thus increases the flow in the pulp [31]. This indicates that under physiological conditions there are no static pressure conditions in the pulp. 
Previous models do not take temperature into account. Investigations are usually carried out at room temperature, which does not correspond to the physiology and can have an influence on the release [18]. In contrast to those investigations our elution experiments were performed at $37^{\circ} \mathrm{C}$. The compactness of the model allowed easy placement within temperature-controlled incubators.

\subsection{Why are residual free monomers present in "photo-cured" dimethacrylate monomers?}

Due to the incomplete conversion of the carbon-carbon double bonds ( $\mathrm{C}=\mathrm{C}$ double bonds) of the monomers during light-induced polymerization, residual monomers remain present in the composite [32]. The deeper the layer, the greater the amounts of residual monomers, i.e. close to the pulp the content is greater than on the outer surface [33]. Due to standardization of the cavity size and thus the size of the composite restoration, the same maximal degree of conversion (DC) of the $\mathrm{C}=\mathrm{C}$ double bonds can be assumed independently of the thickness of the dentin layer, which is under the restoration (and therefore furthest from of the composite surface receiving maximal irradiation). Accordingly, the same amount of residual monomer would have to be present and released. This standardization of the restoration was important to be able to study the kinetics of monomer release in relation to dentin thickness.

\subsection{What can influence the analytical quality, what types of monomer were released and detected and what type of data were generated?}

The analytical quality of a measurement depends not only on the analytics itself but also on the pre-analytics. Many mistakes are made in this area [34]. For example, monomers such as TEGDMA can diffuse into plastics such as reaction vessels or pipette tips and falsify the measurement result (false low) [35]. The stability of the present method was determined by the intra-assay coefficient, analogous to the DIN EN ISO 17189 standard (an International 
Standard for the quality management of medical laboratories). In this context caffeine was used as internal standard. The intra-assay coefficient was below $10 \%$. This value is in good agreement with the usual specifications in this range for this measurement method.

The focus of the analysis was on the detection of bisEMA. Due to the temperature control of the GC/MS and the columns used, this technique could be used to measure both the lowboiling monomers TEGDMA and TEEGDMA and, in separate runs, the high-boiling monomers bisGMA and bisEMA. The amount of eluted substance was determined, as is usual in analysis and independent of the method (GC/MS, HPLC), either by the integrated signal area or by the signal height. Thus only the signal of the analyte was determined, but not yet quantified. To quantify substances in the mass spectrum of GC/MS, either a calibration curve (external standardization) or a labelled (often multiple deuterated) analyte (internal standardization) might be used. Neither options are available for bisEMA. Compared to bisGMA, TEGDMA or TEEGDMA, bisEMA in dental materials is not a single monomer, but rather a generic name for a large homologous series of ethoxylated bisphenol A based dimethacrylate molecules that differ by one ethylene oxide unit (Fig. 2) [19]. The degree of ethoxylation is indicated by the indexes $\mathrm{n}$ and $\mathrm{m}$ (Fig. 2). The respective homologues are not purely available, presumably because of a complex synthesis, labelling and separation of the homologs. Therefore, bisEMA cannot be quantified exactly. However, it is possible to compare the effect of different elution methods or dentin thicknesses by evaluating the signal area or signal height in the mass spectrum. This in turn makes it possible to generate elution kinetics. In order to have a uniform evaluation and easier comparability, all other analytes were also evaluated by this approach.

Only the bisEMA homologous $\mathrm{m}=1$ and $\mathrm{n}=1$ was found in quantifiable quantities in the eluates. In traces, bisEMA homologous up to $n+m=3$ could be found. This is in good agreement with the analysis of the uncured composite dissolved in methanol, where bisEMA 
homologues were found only up to $n+m<4$ in reportable amounts. It is conceivable that with increasing molecule size, i.e. increasing $m+n$, the dentin layer can only be penetrated more slowly, e.g. due to steric effects or electrostatic interactions with the dentin. Besides the above-mentioned increased penetration time through dentin tubuli, it would also be possible that at a certain molecule size no penetration is possible (molecule size vs. diameter of the dentin tubuli).

\subsection{What were the major additional variables considered and what were the major trends?}

Besides the analytes themselves, the release kinetics of the different monomers were analysed. Here, the dentin thickness was also of importance in addition to the time course. With increasing dentin thickness, the amount of bisEMA in the eluate was reduced (6). The dentin layer can act as a diffusion barrier for a molecule the size of bisEMA [36]. However, a diffusion barrier only delays diffusion but does not prevent it. Therefore, different release kinetics had to be evaluated. This was achieved with an exponential (growth to maximum) function (eq. 1) [37]. It was found that the growth constant $\mathrm{k}$ was different for three different ranges of dentin thickness (lower: $1 \mathrm{~mm}$, about $1.5 \mathrm{~mm}$ and higher: $1.8 \mathrm{~mm}$ ). For HEMA, the diffusion quantity as a function of dentin thickness is known and described [38]. For HEMA, the remaining dentin thickness ranges were $0.5-1.9 \mathrm{~mm}$ and $2.0-3.5 \mathrm{~mm}$ [38]. These results, regarding critical residual dentin thickness ranges, for smaller, more hydrophilic molecules such as HEMA, TEGDMA and TEEGDMA do not contradict our present findings. In our study no relevant release of TEGDMA and TEEGDMA could be measured after 24 hours. The cited study examined elution over a shorter period of about 4 min to $24 \mathrm{~h}$ after placing a restoration in 3rd molars. The reasons for these differences from our results with bisEMA may lie in its greater molecule size as well as in its reduced hydrophilicity, which is significantly greater for HEMA, TEGDMA and TEEGDMA [3]. 
As expected, the thinnest dentin thickness showed the lowest kinetic growth constant. This is evidently because the greatest amount of bisEMA was released within the first $24 \mathrm{~h}$ and significantly less elution occurred in the further period of experimental observations. This was different for the thickest dentin layer. There, less elution took place within the first $24 \mathrm{~h}$, which is why more was released in the further period of experimental observations. This was reflected in a higher kinetic growth constant.

After 7 days, at the end of the experiment, the eluate showed less bisEMA in teeth with a thick dentin layer than in teeth with a thin dentin layer after 1 to $2 \mathrm{~d}$. Assuming that a comparable quantity of residual monomers was always present independent of the dentin thickness (due to comparable residual monomer content after photopolymerization: see 4.7 and equivalent restorative procedures), it can be assumed that the elution time was longer through a thick dentin layer. This would explain the clinical experience that some patients have "problems" shortly after cavity restoration, while others develop them only after several days.

Apart from consideration of residual dentin thickness, the selected experimental time course was also important. TEGDMA and TEEGDMA were detectable in the ethanolic-aqueous elution medium independent of the treatment of the teeth over the observational period of 1 to $7 \mathrm{~d}$, mostly in constant quantities (Fig. 4 and 5). This indicates that: (i) the elution of these comonomers should have been completed within $24 \mathrm{~h}$ and (ii) the dentin layer was not a significant diffusion barrier. Similar results were found by Hume et al. [15, 39]. They also showed that the most TEGDMA was elutable within the first day. In their study, human teeth had their roots removed and the stumps closed with wax. They were treated with a defined restoration and then positioned in a polyethylene chamber. In contrast to our experiment, the tooth was not positioned vertically in the elution medium, but horizontally in the chamber. 
Although the hydrostatic conditions would have been different, a similar release rate was determined.

This is also in line with experiments simulating elution into saliva. In some such studies only small amounts were eluted after $24 \mathrm{~h}$ from composites [40] or adhesives [41]. Other studies have shown that TEGDMA elutability was greatest within the first $7 \mathrm{~d}$ [42]. These different data depend, among other parameters, on the different experimental conditions (e.g. irradiation time, composition of the composite).

In the ethanolic aqueous eluates of teeth treated with both the adhesive system and a composite (EW-AC 1-5) greater quantities of TEGDMA and TEEGDMA were not found, compared to teeth treated with only the adhesive system (EW-A 1-5). The polymerized layer of the adhesive may act as a diffusion barrier for TEGDMA and TEEGDMA. However, this, does not explain why bisEMA could diffuse through it. Another possibility would be that by introducing the composite (in our case in a multi-layer technique with multiple light-induced polymerization) further free-radical concentrations were generated which reacted with residual monomers in the adhesive. Residual monomers from the adhesive and the composite, could be eluted within the $7 \mathrm{~d}$ period, which were measured in reduced quantities compared with adhesive treatment alone.

\subsection{What are the results from other studies?}

Mahdhaoui et al. [18] did not find quantifiable amounts of the hydrophobic monomers such as bisGMA in their water-based elution systems of buffered saline without protein. However we did find traces of bisEMA in the water elution medium. In [18], high performance liquid chromatography (HPLC) was used as the analytical method and not GC/MS as in this study. For a long time, high-molecular base monomers such as bisGMA and bisEMA could only be found using HPLC or coupled mass spectrometry (HPLC-MS/MS). The results from our 
present study with an elevated GC temperature showed that the detection of bisGMA and bisEMA (up to a certain number of ethylene oxide units) is also possible using this technique.

The study from Mahdhaoui et al. [18] differed not only in the use of a different analysis technique but also in the dentin model. In contrast to our study, dentin discs were used, with several limitations during the preparation step. In both models, diffusion of HEMA through dentin tubules was shown. But a common feature of both models is that simulated pulpal pressure was not used. Pulpal pressure is usually given as $15 \mathrm{~cm} \mathrm{H}_{2} \mathrm{O}$ [43] and can be simulated in different dentin diffusion models. It is suggested that this pulpal pressure causes a flow of dentin fluid to the oral side e.g. after carious lesion removal and therefore substances could not diffuse from the dentin to the pulp. This is correct, but only if the dentin tubules are open at the oral side, i.e. when no counterpressure is created by a cavity restorative material [18].

The influence of the nature of the dentin (age, caries affection) and its influence on elution and diffusion is discussed in the literature. However, for HEMA, elution studies showed that caries-infested dentin has no statistically significant influence on the diffused amount of HEMA in the eluate compared to healthy dentin [44]. In addition, there are studies that show that permeability is increased or decreased $[45,46]$. One reason for these different results is the extent of the cariogenic defect [44]. The larger the defect, the lower the diffusion barrier for the comonomers HEMA and TEGDMA. The age of the lesion also plays a role [45]. We used caries-free and untreated 3rd molars to exclude these influencing factors. 


\section{Conclusions}

The presented in vitro model showed that sound human $3^{\text {rd }}$ molars, with a cavity preparation and restoration, can be an alternative to dentin discs to simulate the diffusion of monomers through the dentin layer to the pulp. Time-consuming disc preparation steps are no longer necessary.

In this model, a thicker dentin layer resulted in reduced elution kinetics of bisEMA per time interval. In this case, an extended exposure period to the various cells of the pulp could occur. In the selected model, the maximal elution of TEGDMA and TEEGDMA occurred within 24 $\mathrm{h}$ in ethanol/water, so that the concentration within the dental pulp should be greatest during that period. 


\section{References}

[1] Fugolin APP, Pfeifer CS. New Resins for Dental Composites. J Dent Res. 2017;96:1085-91. https://doi.org/10.1177/0022034517720658.

[2] Geurtsen W, Spahl W, Leyhausen G. Variability of cytotoxicity and leaching of substances from four light-curing pit and fissure sealants. J Biomed Mater Res. 1999;44:73-7.

[3] Durner J, Spahl W, Zaspel J, Schweikl H, Hickel R, Reichl FX. Eluted substances from unpolymerized and polymerized dental restorative materials and their Nernst partition coefficient. Dent Mater. 2010;26:91-9. https://doi.org/10.1016/j.dental.2009.08.014.

[4] Wegehaupt FJ, Lunghi N, Belibasakis GN, Attin T. Influence of light-curing distance on degree of conversion and cytotoxicity of etch-and-rinse and self-etch adhesives. BMC Oral Health. 2016;17:12. https://doi.org/10.1186/s12903-016-0239-3.

[5] Putzeys E, Duca RC, Coppens L, Vanoirbeek J, Godderis L, Van Meerbeek B, et al. Invitro transdentinal diffusion of monomers from adhesives. J Dent. 2018;75:91-7. https://doi.org/10.1016/j.jdent.2018.05.023.

[6] Aalto-Korte K, Alanko K, Kuuliala O, Jolanki R. Methacrylate and acrylate allergy in dental personnel. Contact Dermatitis. 2007;57:324-30. https://doi.org/10.1111/j.16000536.2007.01237.x.

[7] Geurtsen W, Lehmann F, Spahl W, Leyhausen G. Cytotoxicity of 35 dental resin composite monomers/additives in permanent 3T3 and three human primary fibroblast cultures. J Biomed Mater Res. 1998;41:474-80. https://doi.org/10.1002/(SICI)10974636(19980905)41:3<474::AID-JBM18>3.0.CO;2-I.

[8] Goldberg M. In vitro and in vivo studies on the toxicity of dental resin components: a review. Clin Oral Investig. 2008;12:1-8. https://doi.org/10.1007/s00784-007-0162-8.

[9] Schweikl H, Spagnuolo G, Schmalz G. Genetic and cellular toxicology of dental resin monomers. J Dent Res. 2006;85:870-7. https://doi.org/10.1177/154405910608501001.

[10] Durner J, Wellner P, Hickel R, Reichl FX. Synergistic interaction caused to human gingival fibroblasts from dental monomers. Dent Mater. 2012;28:818-23. https://doi.org/10.1016/j.dental.2012.04.031.

[11] Lottner S, Shehata M, Hickel R, Reichl FX, Durner J. Effects of antioxidants on DNAdouble strand breaks in human gingival fibroblasts exposed to methacrylate based monomers. Dent Mater. 2013;29:991-8. https://doi.org/10.1016/j.dental.2013.07.005.

[12] Yoon TH, Lee YK, Lim BS, Kim CW. Degree of polymerization of resin composites by different light sources. J Oral Rehabil. 2002;29:1165-73. https://doi.org/10.1046/j.13652842.2002.00970.x.

[13] Gerzina TM, Hume WR. Diffusion of monomers from bonding resin-resin composite combinations through dentine in vitro. J Dent. 1996;24:125-8.

[14] Mjor IA, Odont D. Pulp-dentin biology in restorative dentistry. Part 2: initial reactions to preparation of teeth for restorative procedures. Quintessence Int. 2001;32:537-51.

[15] Gerzina TM, Hume WR. Effect of dentine on release of TEGDMA from resin composite in vitro. J Oral Rehabil. 1994;21:463-8.

[16] Galler K, Hiller KA, Ettl T, Schmalz G. Selective influence of dentin thickness upon cytotoxicity of dentin contacting materials. J Endod. 2005;31:396-9.

[17] Schmalz G, Hiller KA, Nunez LJ, Stoll J, Weis K. Permeability characteristics of bovine and human dentin under different pretreatment conditions. J Endod. 2001;27:2330. https://doi.org/10.1097/00004770-200101000-00007.

[18] Mahdhaoui K, Fournier B, Derbanne MA. Unbound monomers do diffuse through the dentin barrier. Dent Mater. 2017;33:743-51. https://doi.org/10.1016/j.dental.2017.04.007. 
[19] Durner J, Schrickel K, Watts DC, Ilie N. Determination of homologous distributions of bisEMA dimethacrylates in bulk-fill resin-composites by GC-MS. Dent Mater. 2015;31:473-80. https://doi.org/10.1016/j.dental.2015.02.006.

[20] Durner J, Obermaier J, Ilie N. Investigation of different bleaching conditions on the amount of elutable substances from nano-hybrid composites. Dent Mater. 2014;30:1929. https://doi.org/10.1016/j.dental.2013.11.003.

[21] Schneider TR, Hakami-Tafreshi R, Tomasino-Perez A, Tayebi L, Lobner D. Effects of dental composite resin monomers on dental pulp cells. Dent Mater J. 2019. https://doi.org/10.4012/dmj.2018-163.

[22] Schertl P, Volk J, Perduns R, Adam K, Leyhausen G, Bakopoulou A, et al. Impaired angiogenic differentiation of dental pulp stem cells during exposure to the resinous monomer triethylene glycol dimethacrylate. Dent Mater. 2019;35:144-55. https://doi.org/10.1016/j.dental.2018.11.006.

[23] Garberoglio R, Brannstrom M. Scanning electron microscopic investigation of human dentinal tubules. Arch Oral Biol. 1976;21:355-62. https://doi.org/10.1016/s00039969(76)80003-9.

[24] Dourda AO, Moule AJ, Young WG. A morphometric analysis of the cross-sectional area of dentine occupied by dentinal tubules in human third molar teeth. Int Endod J. 1994;27:184-9. https://doi.org/10.1111/j.1365-2591.1994.tb00252.x.

[25] United States Food and Drug Administration (US FDA). Recommendations for chemistry data for indirect food additives petitions. Washington, DC: Food and Drug Administration, Center for Food Safety and Applied Nutrition. USA, 1988.

[26] Moreira Fdo C, Antoniosi Filho NR, Souza JB, Lopes LG. Sorption, solubility and residual monomers of a dental adhesive cured by different light-curing units. Braz Dent J. 2010;21:432-8. https://doi.org/10.1590/S0103-64402010000500010

[27] Cussler EL. Diffusion Mass Transfer in Fluid Systems. 3rd ed. Cambridge: Cambridge University Press; 2009.

[28] Camps J, Tardieu C, Dejou J, Franquin JC, Ladaique P, Rieu R. In vitro cytotoxicity of dental adhesive systems under simulated pulpal pressure. Dent Mater. 1997;13:34-42. https://doi.org/10.1016/s0109-5641(97)80006-1.

[29] de Barros Pinto L, Lira M, Cavalcanti YW, Dantas ELA, Vieira MLO, de Carvalho GG, et al. Natural enamel caries, dentine reactions, dentinal fluid and biofilm. Sci Rep. 2019;9:2841. https://doi.org/10.1038/s41598-019-38684-7.

[30] Puapichartdumrong P, Ikeda H, Suda H. Facilitation of iontophoretic drug delivery through intact and caries-affected dentine. Int Endod J. 2003;36:674-81. https://doi.org/10.1046/j.1365-2591.2003.00712.x.

[31] Su KC, Chuang SF, Ng EY, Chang CH. An investigation of dentinal fluid flow in dental pulp during food mastication: simulation of fluid-structure interaction. Biomech Model Mechanobiol. 2014;13:527-35. https://doi.org/10.1007/s10237-013-0514-z.

[32] Ilie N, Obermaier J, Durner J. Effect of modulated irradiation time on the degree of conversion and the amount of elutable substances from nano-hybrid resin-based composites. Clin Oral Investig. 2014;18:97-106. https://doi.org/10.1007/s00784-0130934-2.

[33] Santos GB, Medeiros IS, Fellows CE, Muench A, Braga RR. Composite depth of cure obtained with QTH and LED units assessed by microhardness and micro-Raman spectroscopy. Oper Dent. 2007;32:79-83.

[34] Durner J. Clinical chemistry: challenges for analytical chemistry and the nanosciences from medicine. Angew Chem Int Ed Engl. 2010;49:1026-51. https://doi.org/10.1002/anie.200903363. 
[35] Durner J, Glasl B, Zaspel J, Kunzelmann KH, Hickel R, Reichl FX. Release of TEGDMA from composite during the chewing situation. Dent Mater. 2010;26:e197204. https://doi.org/10.1016/j.dental.2010.03.003.

[36] Pashley DH, Derkson GD, Tao L, Derkson M, Kalathoor S. The effects of a multi-step dentin bonding system on dentin permeability. Dent Mater. 1988;4:60-3.

[37] Ferrell N, Desai RR, Fleischman AJ, Roy S, Humes HD, Fissell WH. A microfluidic bioreactor with integrated transepithelial electrical resistance (TEER) measurement electrodes for evaluation of renal epithelial cells. Biotechnol Bioeng. 2010;107:707-16. https://doi.org/10.1002/bit.22835.

[38] Tak O, Usumez A. Diffusion of HEMA from resin cements through different dentin thicknesses in vitro. Am J Dent. 2015;28:285-91.

[39] Hamid A, Hume WR. The effect of dentine thickness on diffusion of resin monomers in vitro. J Oral Rehabil. 1997;24:20-5.

[40] Ferracane JL, Condon JR. Rate of elution of leachable components from composite. Dent Mater. 1990;6:282-7. https://doi.org/10.1016/S0109-5641(05)80012-0.

[41] Miletic V, Santini A, Trkulja I. Quantification of monomer elution and carbon-carbon double bonds in dental adhesive systems using HPLC and micro-Raman spectroscopy. J Dent. 2009;37:177-84. https://doi.org/10.1016/j.jdent.2008.11.006.

[42] Polydorou O, Konig A, Hellwig E, Kummerer K. Long-term release of monomers from modern dental-composite materials. Eur J Oral Sci. 2009;117:68-75. https://doi.org/10.1111/j.1600-0722.2008.00594.x.

[43] Ciucchi B, Bouillaguet S, Holz J, Pashley D. Dentinal fluid dynamics in human teeth, in vivo. J Endod. 1995;21:191-4. https://doi.org/10.1016/S0099-2399(06)80564-9.

[44] Tak O, Usumez A. Diffusion of HEMA through human carious and non-carious dentin in vitro. J Prosthodont. 2013;22:184-9. https://doi.org/10.1111/j.1532849X.2012.00935.X.

[45] Tagami J, Hosoda H, Burrow MF, Nakajima M. Effect of aging and caries on dentin permeability. Proc Finn Dent Soc. 1992;88 Suppl 1:149-54.

[46] Hamid A, Hume WR. Diffusion of resin monomers through human carious dentin in vitro. Endod Dent Traumatol. 1997;13:1-5. 


\section{Figure captions}

Figure 1. Schematic presentation of the experimental setup. In a healthy tooth, an artificial cavity was treated by a standardised procedure. In order to investigate whether substances from the restorative material can diffuse through the (residual) dentin and thus reach the pulp, the tooth root was coated with elution medium up to the base of the tooth crown. Substances from the restorative material, which are detected in the elution medium, must have diffused through the dentin tubules and can also reach the pulp in this way.

Figure 2. Chemical structure of (a) ethoxylated bisphenol A dimethacrylate (bisEMA), $\mathbf{n}$ and $\mathbf{m}$ are the numbers of repeats of the ethoxy unit, (b) bisphenol A glycidyldimethacrylate (bisGMA), (c) triethylene glycol dimethacrylate (TEGDMA) and (d) tetraethylene glycol dimethacrylate (TEEGDMA)

Figure 3. Elution kinetics for triethyleneglycol dimethacrylate (TEGDMA) and tetraethyleneglycol dimethacrylate (TEEGDMA) after treatment of the teeth with an adhesive system and a composite. The elution medium was water. The plots show the integrated signal area as a function of time. In addition, the reference of the area to the dentin thickness is shown.

Figure 4. Elution kinetics for triethyleneglycol dimethacrylate (TEGDMA) and tetraethyleneglycol dimethacrylate (TEEGDMA) after treatment of the teeth with an adhesive system. The elution medium was ethanol/water (3:1). The plots show the integrated signal area as a function of time. In addition, the reference of the area to the dentin thickness is shown.

Figure 5. Elution kinetics for triethyleneglycol dimethacrylate (TEGDMA) and tetraethyleneglycol dimethacrylate (TEEGDMA) after treatment of the teeth with an adhesive system and a composite. The elution medium was ethanol/water (3:1). The plots show the integrated signal area as a function of time. In addition, the reference of the area to the dentin thickness is shown.

Figure 6. Elution kinetics from ethoxylated bisphenol A dimethacrylate (bisEMA) after treatment of the teeth with an adhesive system and a composite. The elution medium was ethanol/water 3:1. The plots show the integrated signal area as a function of time. In addition, the reference of the area or height to the dentin thickness is shown. 
Figure 1

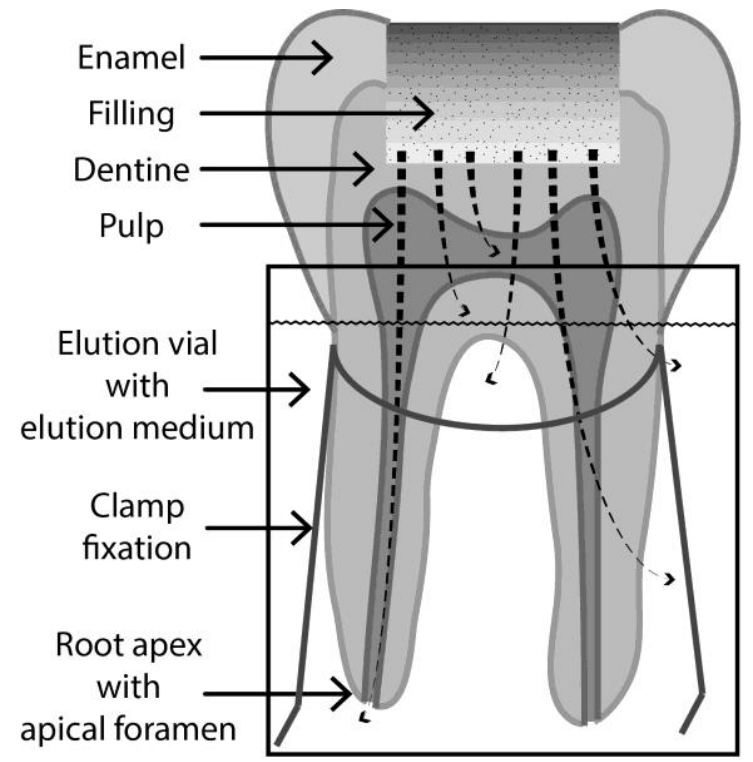


Figure 2

a)

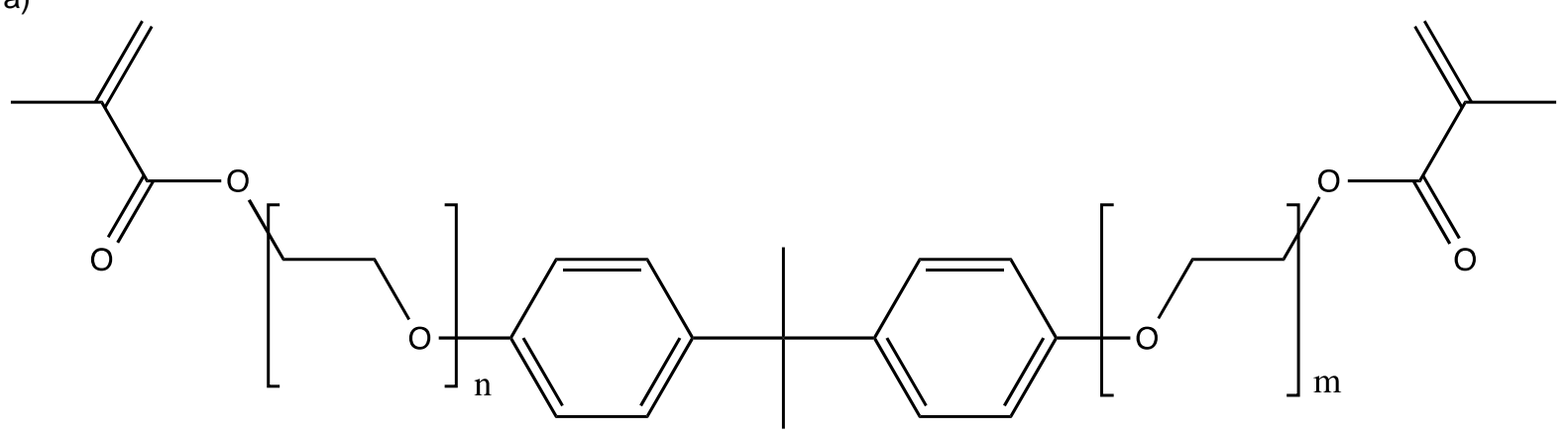

b)<smiles>C=C(C)C(=O)OCC(O)COc1ccc(C(C)(C)c2ccc(OCC(O)COC(=O)C(=C)C)cc2)cc1</smiles>

c)

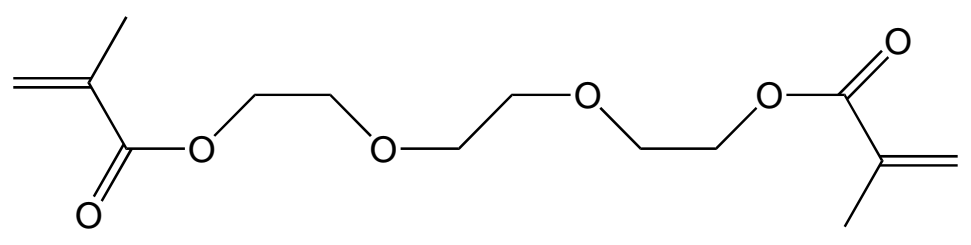

d)<smiles>C=C(C)C(=O)OCCOCCOCCOCCOC(=O)C(=C)C</smiles> 
Figure 3

TEGDMA and TEEGDMA (Area) in aqueous eluates

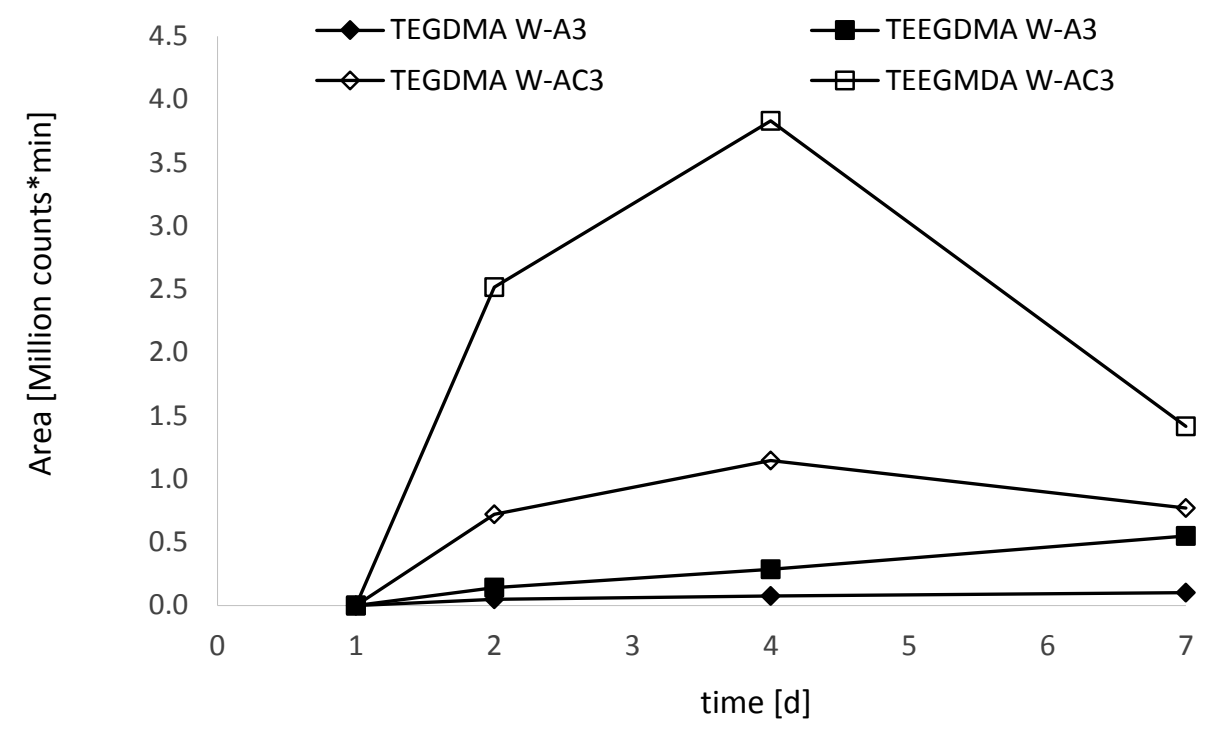

TEGDMA and TEEGDMA (Area) in aqueous eluates/Dentin Thickness

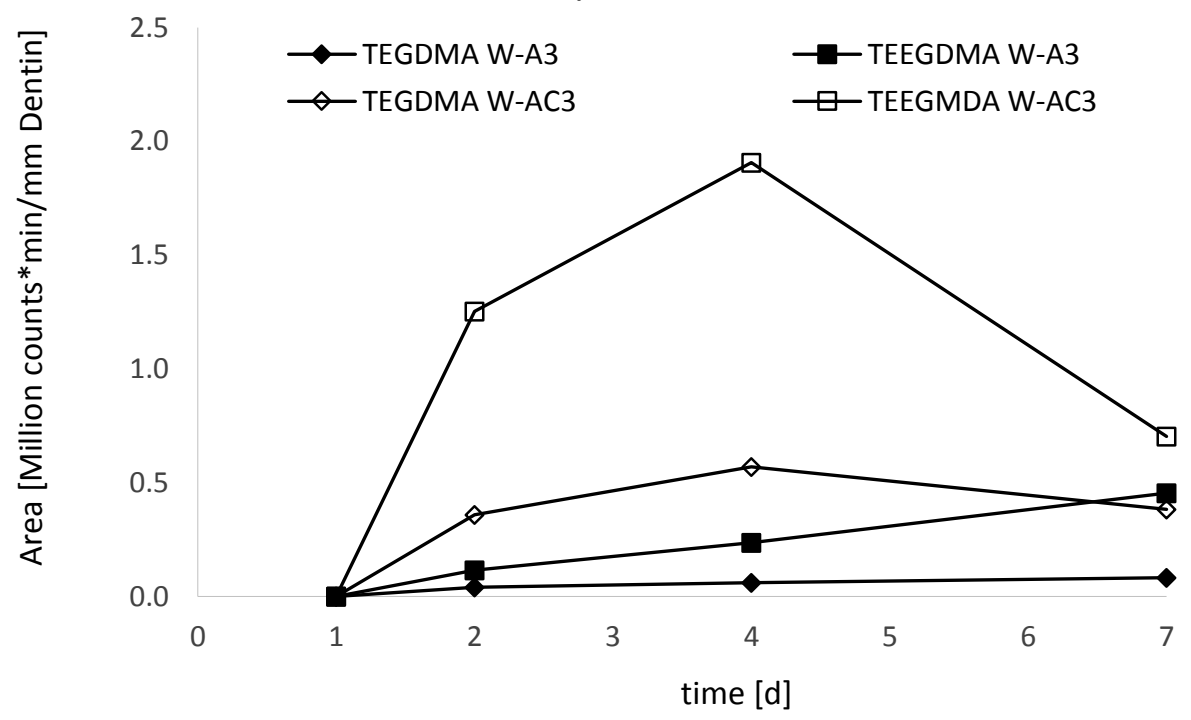


Figure 4

TEGDMA (Area)

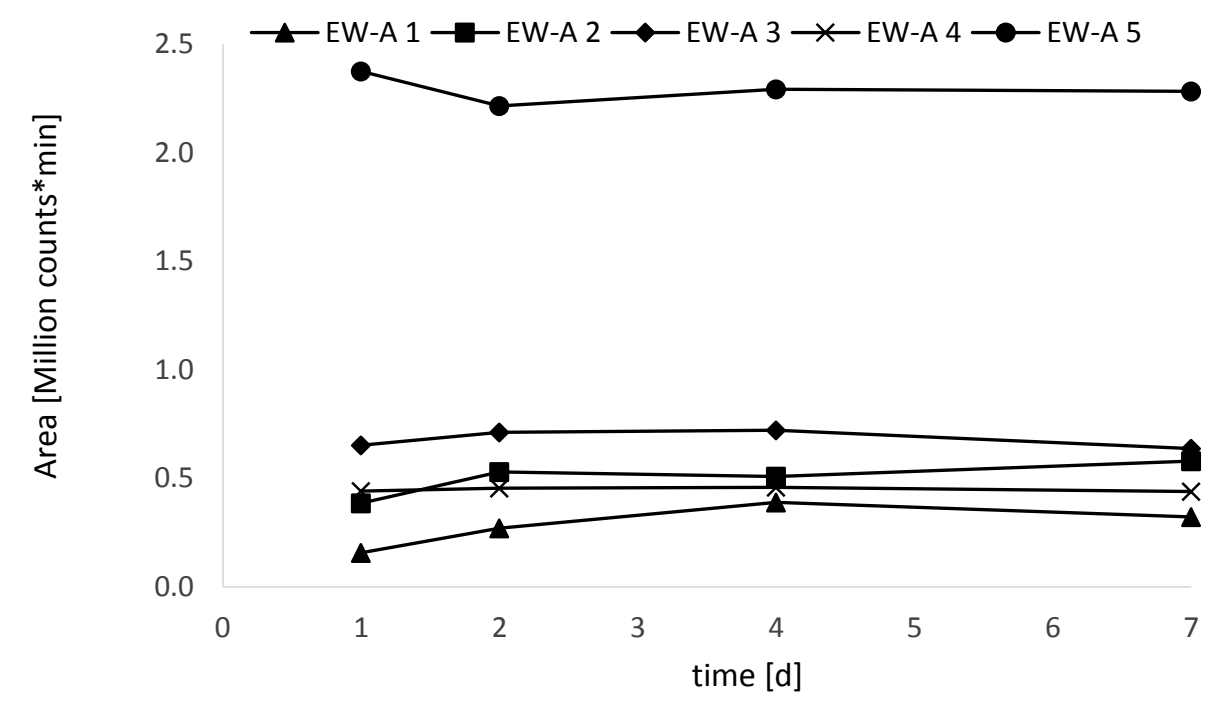

TEEGDMA (Area)

$35 \rightarrow$ EW-A $1 \rightarrow$ EW-A $2 \multimap$ EW-A $3 \rightarrow$ EW-A $4 \longrightarrow$ EW-A 5

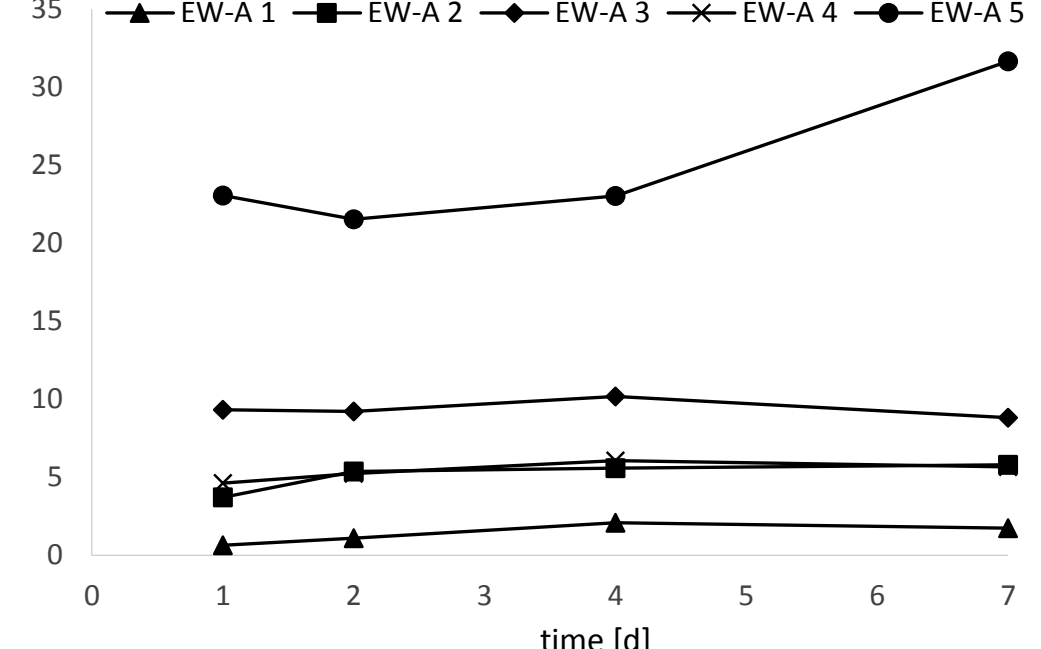

TEGDMA (Area)/Dentin Thickness

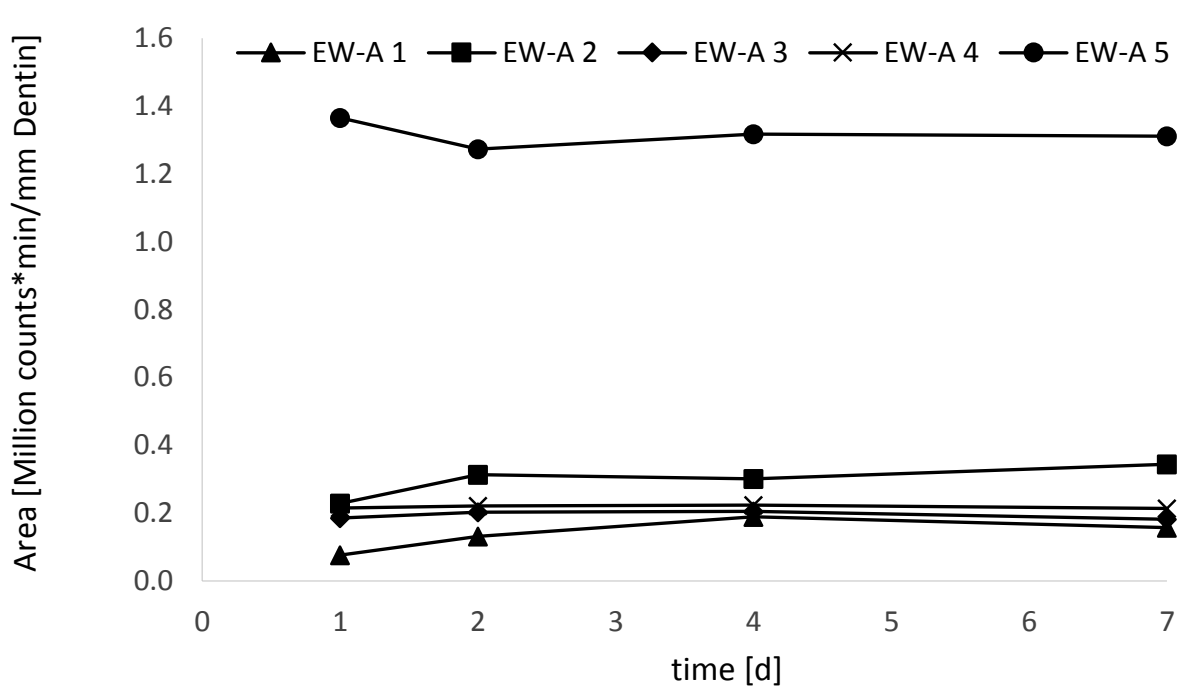

TEEGDMA (Area)/Dentin Thickness

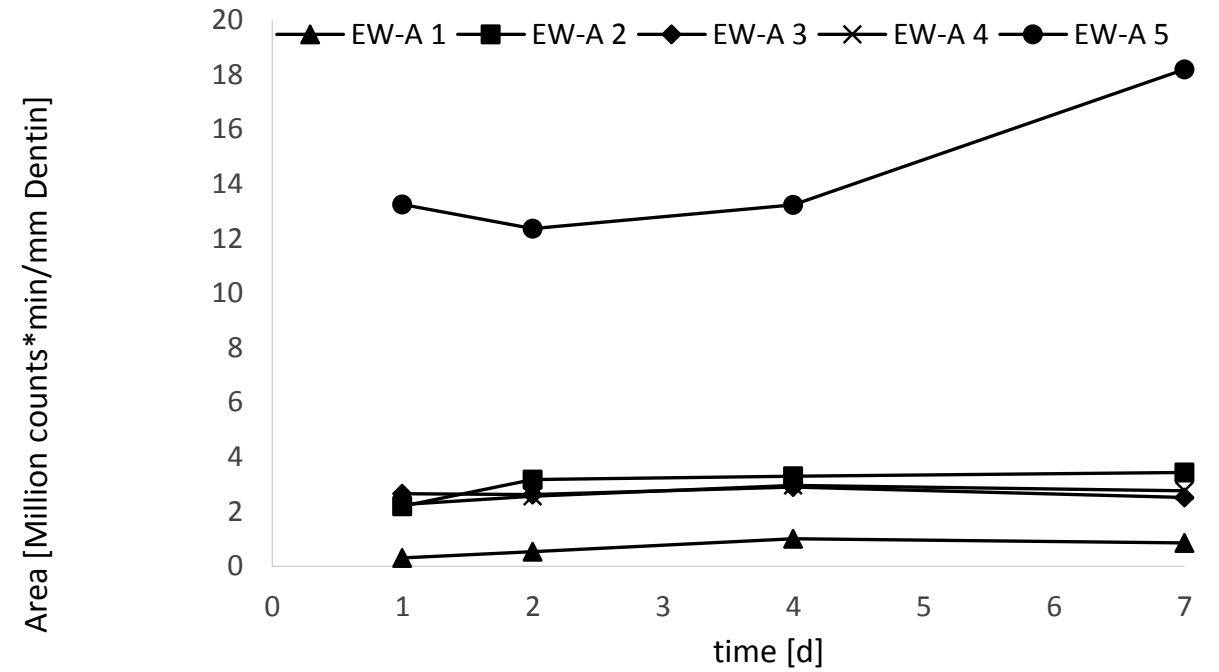


Figure 5

TEGDMA (Area)

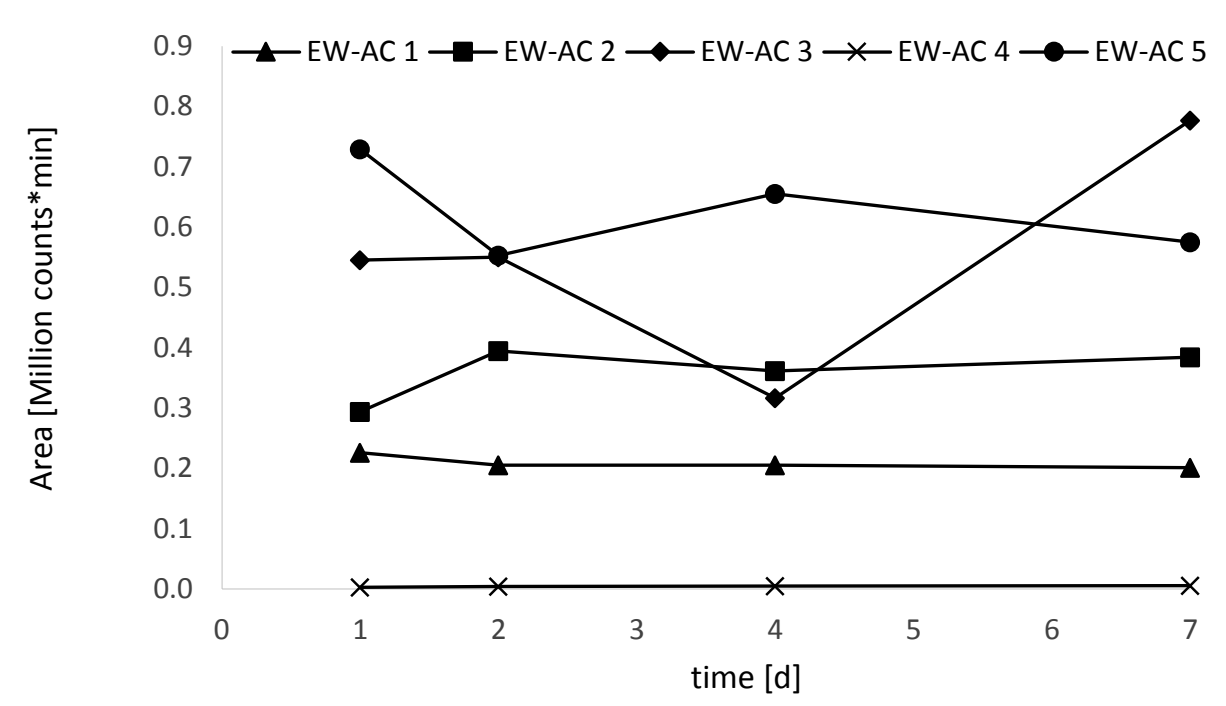

TEEGDMA (Area)

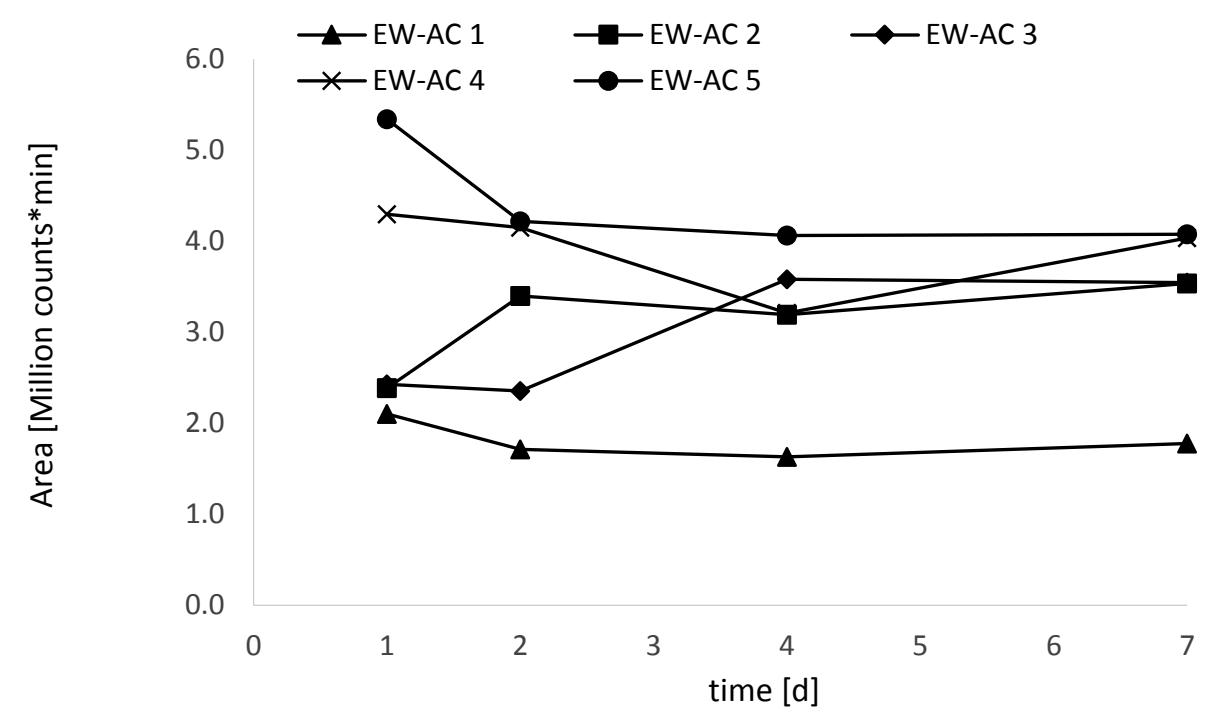

TEGDMA (Area)/Dentin Thickness

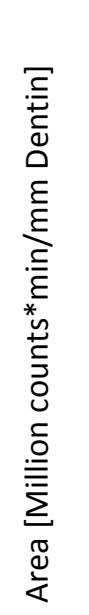

0.8

0.7

0.6

0.5

0.4

0.3

0.2

0.1

0

0 $\neg$ EW-AC $1 \rightarrow$ EW-AC $2 \multimap$ EW-AC $3 \rightarrow$ EW-AC $4 \multimap$ EW-AC 5

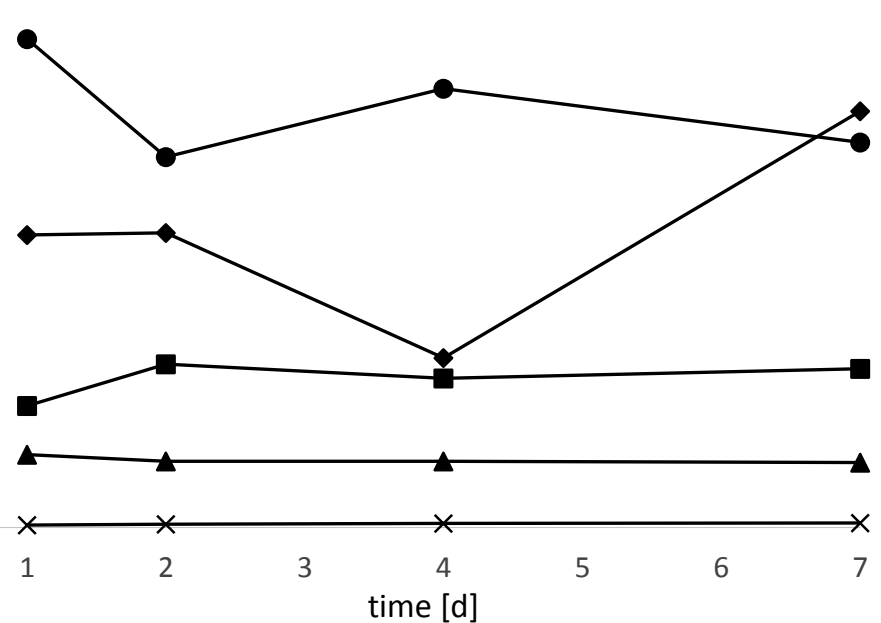

TEEGDMA (Area)/Dentin Thickness

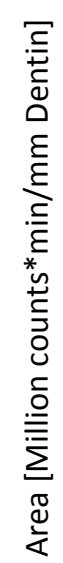

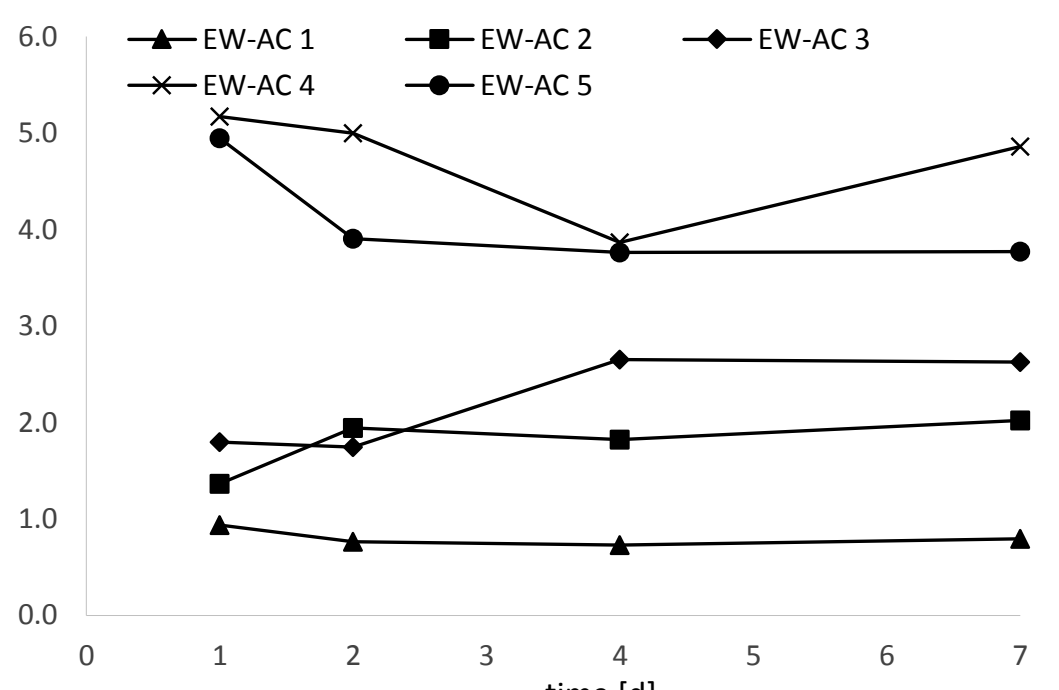


Figure 6

$$
\text { bisEMA (Area) }
$$

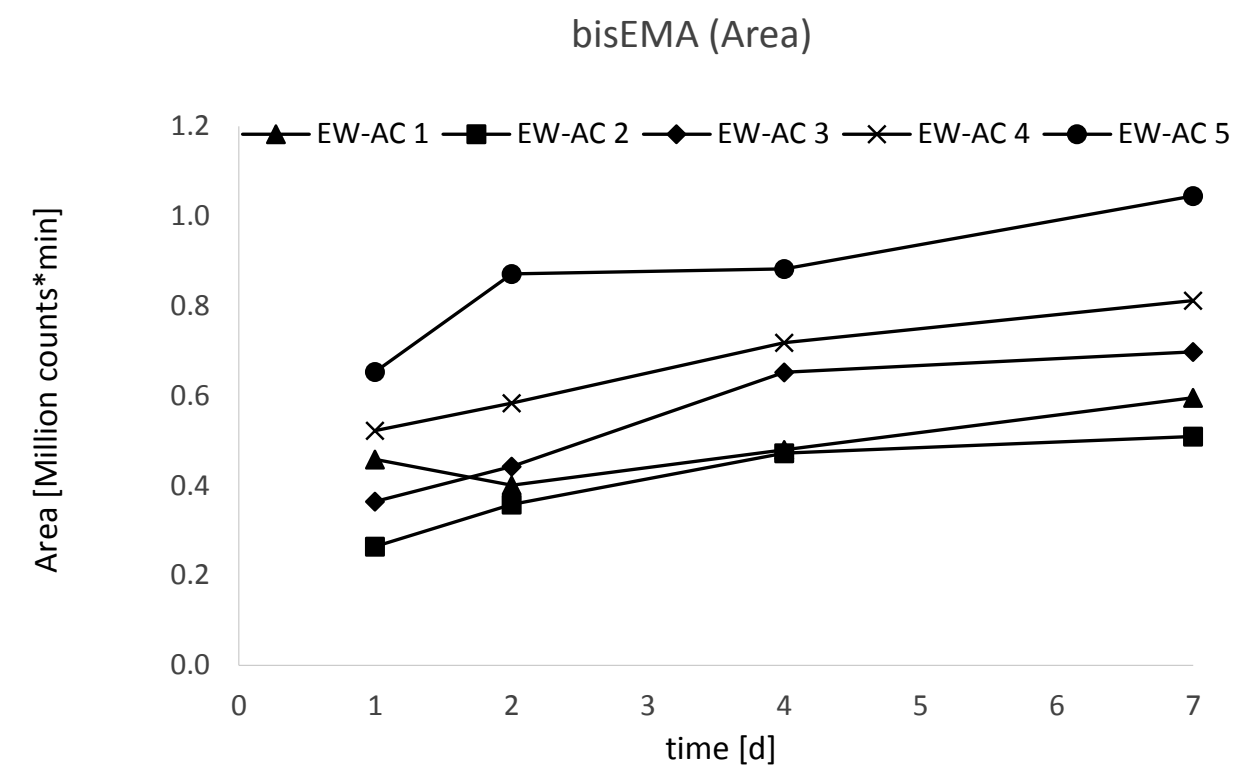

bisEMA (Area)/Dentin Thickness

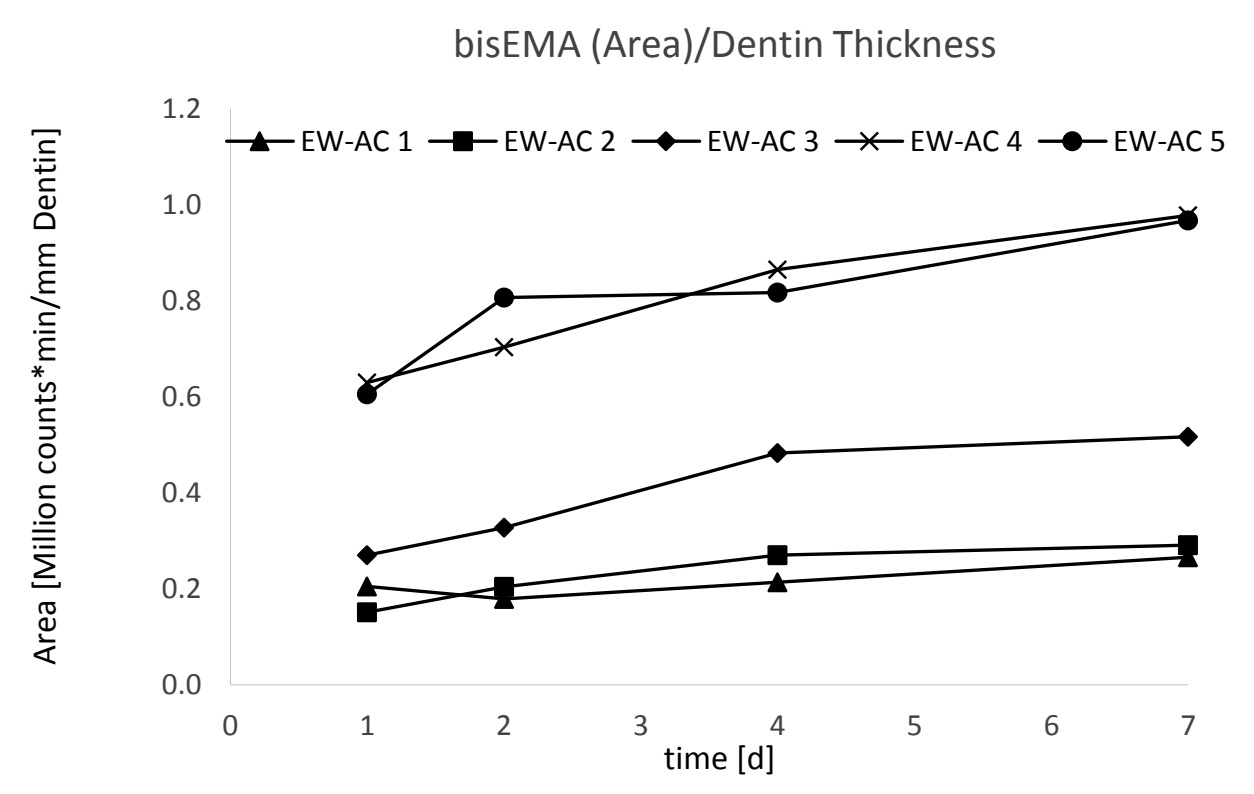


Click here to access/download

Table

Table 1.docx 
Table 2

Click here to access/download

Table

Table 2.docx 